Meta

Journal des traducteurs

Translators' Journal

\title{
Traduisibilité et intraduisibilité
}

\section{Ludwig Söll}

Volume 16, numéro 1-2, mars 1971

Actes du colloque international de linguistique et de traduction.

Montréal, 30 septembre - 3 octobre 1970

URI : https://id.erudit.org/iderudit/004199ar

DOI : https://doi.org/10.7202/004199ar

Aller au sommaire du numéro

Éditeur(s)

Les Presses de l'Université de Montréal

ISSN

0026-0452 (imprimé)

1492-1421 (numérique)

Découvrir la revue

Citer cet article

Söll, L. (1971). Traduisibilité et intraduisibilité. Meta, 16(1-2), 25-31.

https://doi.org/10.7202/004199ar d'utilisation que vous pouvez consulter en ligne.

https://apropos.erudit.org/fr/usagers/politique-dutilisation/ 


\section{Traduisibilité et intraduisibilité}

Traduisibilité et intraduisibilité sont les deux pôles opposés qui délimitent la science de la traduction et entre lesquels se situe l'activité effective du traducteur dans toute sa grandeur et sa misère. Les points de vue extrêmes présupposent des conceptions divergentes de la langue.

Lorsque, par exemple, dans la théorie sur le contenu de la langue (la Sprachinhaltsforschung) établie par Leo Weisgerber, qui part de W. von Humboldt et a nombre de points communs avec la thèse dite de Sapir-Whorf, lorsque donc la langue implique une vision du monde qui lui est propre, traduire constitue plus qu'une métamorphose au niveau de la langue, plus qu'une transposition dans un autre code : la traduction est alors une métamorphose spirituelle et par suite ne peut plus être considérée comme une traduction à proprement parler, car au changement de code est nécessairement liée une modification de l'information transmise. Je citerai comme ouvrage typique de cette tendance le livre de Helmut Gipper, Sprachliche und geistige Metamorphosen bei Gedichtübersetzungen. Eine sprachvergleichende Untersuchung : zur Erhellung deutsch-französischer Geistesverschiedenheit [Métamorphoses linguistiques et spirituelles dans les traductions de poèmes. Une recherche de linguistique comparée visant à mettre en lumière la différence d'esprit entre l'allemand et le français] (Düsseldorf, 1966). L'accent y est mis sur la «métamorphose spirituelle », comme le sous-titre l'indique déjà. À mon avis Gipper n'a pas atteint son but ${ }^{1}$ et ses «métamorphoses spirituelles » sont tout au plus des «métamorphoses poétiques» et ne concernent donc pas «la différence d'esprit entre le français et l'allemand» mais la différence «spirituelle » entre des poèmes et leurs traductions. Le problème de la divergence n'est plus un problème linguistique, mais esthétique, qui a cependant, comme nous allons le voir, des origines spécifiquement liées à la langue. Gipper a donc bien réussi à montrer l'intraduisibilité, mais pas l'intraduisibilité en tant que telle entre les langues, seulement l'intraduisibilité entre des produits bien précis de ces langues.

À la conception de la langue liée au contenu en tant que vision du monde s'oppose la conception liée à la forme, qui voit dans la langue principalement un instrument de l'esprit humain servant essentiellement à la communication. Certes

1. Cf. également le compte rendu de Gottfried Kolde dans : Indogermanische Forschungen, 74 (1969), p. 185-191. 
l'information est transmise sous une certaine forme, mais cette forme n'atteint pas la pensée en tant que telle, elle est dans une certaine mesure structurée en surface et non pas structurée en profondeur. Il ne fait aucun doute que cette conception domine largement de nos jours dans la linguistique et la science de la traduction. C'est par exemple la conception de la grammaire générative-transformationnelle qui présuppose l'existence d'universaux formels profonds et implique par là que toutes les langues sont constituées sur le même modèle, sans postuler pour autant une correspondance point par point entre les diyerses langues, et qui fait en outre entrer en ligne de compte l'existence d'universaux substantiels et tient compte des informations non linguistiques entrant dans le processus de la traduction ${ }^{2}$. Manfred Bierwisch oppose résolument à la thèse du relativisme linguistique issue de Whorf et Weisgerber l'hypothèse des universaux, et postule comme composante nécessaire de la théorie de l'apprentissage du langage (cela vaut aussi pour la théorie de la traduction) un système d'universaux linguistiques duquel résulte une prédisposition universelle des structures de pensée ${ }^{3}$. Ainsi, il est possible, pour donner un exemple souvent cité, de traduire la riche terminologie de la langue des Esquimaux concernant l'eau gelée dans toute autre langue avec tout degré d'exactitude désiré, même si cela nécessite parfois des moyens très importants, parce que la constitution de lexèmes par des complexes de traits sémantiques constitue un universel formel. La traduisibilité ne trouve ses limites que là où commence l'ignorance des choses (laquelle n'est pas obligatoirement due à des différences de culture). Ce qui n'existe que chez les Esquimaux ne peut être traduit directement en français, etc. Mais ce n'est pas là un problème à proprement parler linguistique ni, assurément, un argument pour étayer la théorie de la langue comme vision du monde. D'une part la chose inconnue ou le concept inconnu peuvent être rendus saisissables au moyen de la langue, grâce à un commentaire, peuvent être pour ainsi dire constitués par l'intermédiaire de la langue, d'autre part ces difficultés de compréhension n'existent pas seulement entre les différentes langues, mais aussi à l'intérieur d'une même langue : un Français non skieur doit aussi se faire expliquer les termes : la poudreuse, la folle, la tôle d'hiver, la croûte de vent, la crô̂te lisse, etc. ${ }^{4}$, auquel cas la motivation linguistique des signes peut assurément être une aide, mais tout aussi bien un piège. La traduisibilité absolue de tout contenu linguistique est en fin de compte un dogme de la théorie marxiste de la traduction. Ainsi O. Kade constate ${ }^{5}$ : « Si nous partons de l'idée que tout peut être exprimé dans toute langue, on peut en conclure que, en principe, tout peut être traduit de toute langue donnée dans toute autre langue donnée. $\gg$ Ceci valant d'ailleurs surtout pour la traduction pragmatique, c'est-à-dire la traduction de textes non littéraires.

Les pôles extrêmes de l'intraduisibilité et de la traduisibilité fondamentales seraient ainsi caractérisés, cette dernière semblant correspondre à l'état actuel des connaissances en linguistique, encore que cet éternel point de litige appelle

2. Cf. Noam Chomsky, Aspects of the Theory of Syntax, MIT, 1965, chap. I, paragr. 5.

3. Manfred Bierwisch, "Strukturalismus. Geschichte, Probleme und Methoden ", Kursbuch, 5 (1966), p. 77-152, particulièrement p. 135-137.

4. Cf. Georges Mounin, les Problèmes théoriques de la traduction, Paris, 1963, p. 193.

5. Zufall und Gesetzmäßigkeit in der Übersetzung, Leipzig, 1968, p. 68. Cf. aussi Werner Koller, "Eine linguistische Theorie des Übersetzens", Moderna Språk, 63 (1969), p. 264-270. 
sans doute une réponse plus différenciée, ce qui ressort déjà du fait que la plupart des théoriciens veulent que l'on considère leurs solutions comme valables «en principe ». Dans la pratique les traductions ne sont bien souvent pas aussi adéquates (et ne peuvent l'être) que le voudrait la théorie. Du Bellay l'avait déjà compris et exprimé par un «je ne sais quoi», qu'il convient d'objectiver ${ }^{6}$ : «... chacune Langue a je ne scay quoi propre seulement à elle, dont si vous efforcez exprimer le naif en une autre Langue, observant la loy de traduyre, qui est n'espacier point hors des limites de l'aucteur, vostre diction sera contrainte, froide, et de mauvaise grace. $\gg$

Il semble qu'une réponse différenciée pourrait être apportée à cette éternelle question à l'aide des catégories de substance, de forme, d'expression et de contenu distinguées par Hjelmslev. En ce qui concerne la substance, la substance de l'expression ne concerne ni la linguistique ni la communication et n'est donc jamais un problème de traduction. Par contre, conserver la substance du contenu constitue l'essentiel de toute traduction et la condition de l'équivalence. Or, il ne semble pas que l'on ait démontré ni que l'on puisse démontrer que l'intraduisibilité principielle se situe dans ce domaine. C'est le domaine des structures profondes universelles, de l'absolu de l'expression, et donc également, puisque aucune structure superficielle spécifique de la langue n'est impliquée, de l'absolu de la traduction. Mais cette constatation ne peut se rapporter qu'au que et non pas au comment de 1'《exprimabilité $»$ et de la traduisibilité. Des critères tels que ceux d'économie, d'efficacité, d'esthétique de la réalisation par la langue ne sauraient être introduits ici. Les questions de forme jouent un rôle tout aussi réduit, car elles concernent les structures superficielles et donc la diversité du langage humain : il n'est pas de texte, même de texte traduit, qui puisse faire partie de plus d'un système linguistique. Il convient en outre de préciser que la distinction radicale entre structures profondes et structures superficielles, entre substance et forme implique également que la nature de la segmentation formelle soit tout aussi insignifiante que la forme en soi. On ne peut donc juger de l'équivalence substantielle des contenus que d'après l'ensemble du texte, d'après la communication totale. Les composantes du texte (phonèmes, morphèmes, mots syntagmes, phrases) ne sont pas par elles-mêmes des communications totales et n'ont donc pas besoin de se correspondre au niveau du contenu, ce qu'en général elles ne font d'ailleurs pas. Une équivalence typologique est tout aussi peu nécessaire : un morphème peut aussi être rendu par un mot, etc.

Or, il peut arriver que dans un texte, dans une communication, des traits formels soient essentiels pour la communication. L'objet de la communication n'est plus alors la seule substance, mais aussi la forme, non plus seulement les structures profondes, mais aussi les structures superficielles pour ainsi dire. La langue n'est plus seulement un moyen, mais aussi un but. Cela vaut non seulement pour la langue poétique, la poésie n'étant que le lieu où cette réification de la langue est la plus dense. La faculté de s'élever au-dessus de la langue, d'aller au-delà de la langue est une faculté commune à tous les hommes, l'homme est un être doué non seulement de langage mais aussi de métalangage. Alors, les

6. Joachim du Bellay, la Deffence et illustration de la langue françoyse, édition critique Henri Chamard, STFM, Paris, 1948, p. 36. 
formes à la place des substances ne peuvent plus être traduites, au sens courant de ce terme, l'intraduisibilité commence exactement là où la forme devient un élément signifiant (des équivalences formelles fortuites entre les langues ne changent rien aux problèmes fondamentaux). Qu'il s'agisse dans ce cas de la forme de l'expression ou de la forme du contenu ne joue aucun rôle. Il serait tout au plus possible que les valeurs d'expression formelles, qui produisent d'ailleurs leur effet moins quand elles sont isolées que quand elles sont combinées entre elles, soient moins stables au niveau de l'intersubjectivité (que l'on pense ici à l'onomatopée ou à la symbolique des sons) que les valeurs de contenu formelles (par exemple le jeu de mots).

L'idée que la langue en tant qu'objet devient un problème de traduction généralement insoluble n'est pas précisément nouvelle ${ }^{7}$. Récemment, E. Coseriu y a de nouveau fait référence ${ }^{8}$. "Mais d'un autre côté, la langue appartient aussi à la réalité. Dans un texte, on peut donc aussi utiliser la langue comme phénomène récl et discourir sur la langue. Quand cela se produit, il est évident que la langue ne peut plus être traduite. » Admettons donc que ce fait soit reconnu et assuré, et qu'il marque très nettement la frontière entre la traduisibilité et l'intraduisibilité. Devons-nous en rester là ? Je ne le pense pas. Il semble peu satisfaisant d'élargir la notion de traduction qui se situe au niveau de la langue à des procédés situés au niveau de la métalangue. On devrait bien plutôt introduire des notions différentes et placer à côté de la traduction la métatraduction. Il se révèle alors immédiatement que la frontière entre traduisibilité et intraduisibilité recoupe celle qui sépare langue et métalangue, traduction et métatraduction. Or la métatraduction est tout aussi possible que la traduction parce que la réification du langage, même si elle se produit nécessairement de façon différente dans les différentes langues, est un universel tout comme la structuration profonde. Toutes les langues connaissent les jeux de sonorité et de mots, dans toute langue, la langue elle-même peut se refléter. C'est pourquoi tous les jeux de langue (j'utilise ce terme pour désigner la langue en tant qu'objet de communication) peuvent être rendus non pas par une traduction, mais par une métatraduction. Pour donner un exemple : la communication « Tu as oublié la viande » se traduit sans peine par $D u$ hast das Fleisch vergessen, sans que le fait que viande ait une autre signification linguistique que Fleisch (chair se traduirait aussi par Fleisch) joue ici aucun rôle, car les significations actualisées dans le discours et la chose envisagée sont identiques. Il en est autrement pour la métacommunication (communication qui concerne une langue et non pas un état de choses, une chose) : « Tu as confondu chair et viande ». Ici seule une métatraduction est possible : Du hast chair und viande verwechselt ou bien Du hast die Wörter für «lebendes Fleisch» und «Fleisch zum Verzehr » verwechselt. Mais cette métatraduction est une restitution tout à fait valable, une traduction adéquate au sens large, qui peut être poussée au degré de précision désiré et parfaitement comprise : dans son cadre métalinguistique, mais cela va de soi.

Le caractère unique d'une langue ne dépendant pas seulement de ses

7. Cf. J. C. Catford, $A$ Linguistic Theory of Translation, Londres, 1965.

8. Dans Sprachwissenschaft und Übersetzen, Commentationes Societatis Europaeae III, Munich, 1970, p. 120. 
structures superficielles, mais aussi de l'unicité de son histoire et de l'unicité de sa stratification en registres divers, d'autres problèmes de traduction se posent qui ne peuvent être résolus eux aussi que par une métatraduction. Le recours conscient, par exemple, à des moments passés de l'évolution de la langue (par l'emploi de certains archaïsmes ou de citations dont la forme seule est déjà une allusion au passé) ne peut être rendu per se dans une autre langue, mais peut l'être par analogie, car chaque langue, même quand elle ne possède pas de tradition littéraire, connaît l'historicité en tant que telle. Ou pour m'exprimer autrement : la langue en tant que energeia et non pas seulement en tant que ergon est aussi un universel. Cela se manifeste très communément dans l'aspect créateur de l'activité linguistique du sujet parlant, dans sa capacité d'invention linguistique, capacité à laquelle un élément historique est par définition inhérent. La mesure dans laquelle le caractère d'energeia de la langue permet, dans chaque cas particulier, de reproduire le discours originel (réalisation de la métatraduction en tant que traduction), dépend de la capacité individuelle d'invention linguistique, et c'est là une question qui concerne non plus la théorie mais la pratique de la traduction, non plus la traduction en tant que science, mais la traduction en tant qu'art.

Le même problème se pose mutatis mutandis en ce qui concerne l'étagement des niveaux de langue. Tout locuteur dispose de plus d'un système linguistique, il a à sa disposition des registres de langue géographiques et socioculturels dont il peut faire un usage stylistique. Considérant cette situation A. J. Greimas va si loin qu'il déclare ${ }^{9}$ : « un texte, lorsqu'il se présente en une langue naturelle, peut relever, et relève toujours, de plusieurs systèmes à la fois $\gg$. Il est évident que les constellations linguistiques qui en résultent ne sont pas immédiatement et directement traduisibles, mais ces constellations sont communicables. C'est encore un problème de pratique et d'art de la traduction de tâcher de rendre le plus saisissables possible les effets de langue qui en découlent, par exemple par la substitution de variantes diatopiques ou diastratiques.

La problématique de la traduction littéraire n'est pas le véritable objet de cette communication. Mais il convient de noter qu'une théorie de la traduction littéraire doit reposer sur une théorie générale de la traduction. Elle me semble devoir comporter deux composantes essentielles : a) La traduction littéraire se heurte toujours justement aux problèmes de la métatraduction ; b) La traduction littéraire est en outre toujours la transposition non seulement d'une information tout court mais encore d'une information visant à produire un effet, d'une information «esthétisée 》.

Or la réalisation d'une métatraduction est en principe toujours possible, comme nous l'avons montré, mais on ne peut y parvenir au niveau de la traduction, si tant est que cela soit possible, qu'avec une perte d'effectivité. Ce qu'il y a d'unique dans une langue (qua langue) ne peut être immédiatement reproduit. Cette première perte est encore accrue par l'autre perte, la perte «esthétique 》, qui est inévitable dans la mesure où tout est certes traduisible, mais pas avec le même degré de densité. Une théorie strictement linguistique de la traduction

9. * Pour une sociologie du sens commun », Revue romane, IV, 2 (1969), p. 129-137, particulièrement p. 129. 
doit considérer la paraphrase comme équivalente (la paraphrase est constitutive de tout ce qui concerne le langage), la théorie de la traduction littéraire par contre ne peut la considérer que comme ersatz et de ce fait comme non équivalente. Pratiquement cela signifie que, comme on le sait depuis longtemps, les poèmes sont intraduisibles en tant qu'œuvres d'art et par là même absolument intraduisibles, car un poème n'est poème qu'en tant qu'œuvre d'art ${ }^{10}$. L'art du traducteur ne peut jamais atteindre ici l'équivalence, comme c'est le cas pour la traduction pragmatique, mais seulement la reproduction (reproduction dans le sens non de répétition mais d'imitation ${ }^{11}$ ). Ceci semble fixer les limites d'une théorie générale de la traduction, mais ces limites ne concernent à la vérité ni la traduisibilité ni les langues en tant que systèmes, mais le langage en tant qu'activité créatrice. Aucune formulation linguistique originale ne peut être totalement transposée, que ce soit à l'intérieur d'une même langue ou d'une langue dans l'autre. Plus même : si nulle traduction ne peut être parfaite du point de vue esthétique, il convient de dire également qu'aucun texte (écrit ou oral) dans sa propre langue ne peut être parfait en soi (comme réalisation totale de la langue) mais seulement par soi (comme accomplissement individuel de la langue). Dans cette mesure, l'insuffisance de fait de la traduction même au niveau littéraire ne dément pas la traduisibilité principielle. La théorie de la traduction ne peut s'étendre qu'au domaine de validité intersubjectif de la langue, mais ne peut pénétrer dans la subjectivité du locuteur. Ce qu'il y a d'unique dans une formulation littéraire a des limites qui tiennent non pas à la langue mais au locuteur; se mettre d'accord sur un poème est un problème qui se pose non seulement de langue à langue mais aussi au sein d'une même langue. Toujours est-il que la possibilité d'une recréation de langue à langue, quelles que soient ses chances, subsiste du fait de la subjectivité du langage et pas seulement des langues, du fait du caractère ouvert du langage et des langues, ce qui constitue également un universel. Une telle transposition créatrice dans une même langue ou de langue à langue n'est ni programmable ni prévisible. C'est pourquoi la machine à traduire (MT) ne pourra jamais remplacer la traduction humaine (HT).

LUDWIG SöLL

\section{QUESTIONS}

M. Lacoursière : Quand vous parlez de traduisibilité, vous parlez de degré de difficulté ? Est-ce que, si j'ai bien compris, le degré d'abstraction du texte à traduire crée la difficulté du texte de traduction?

Réponse : Si vous me le permettez, j'aimerais choisir l'exemple du passif. Normalement, je dirais que tout passif est traduisible, dans un texte pragmatique, certainement. Il peut y avoir des problèmes d'équivalence, d'équivalence stylistique si, par exemple, on traduit plutôt un passif anglais par une construction avec " on " en français. Mais en principe, il est possible de constituer, d'atteindre une équivalence totale parce qu'il ne s'agit pas ici de traduire une certaine forme forme du passif, forme de la construction avec " on * - mais l'idée, l'élément de structure profonde qui est commun à toutes les langues. Donc normalement, aucun problème. Mais il pourrait arriver que dans un texte, soit le passif anglais, soit la construction avec " on " en

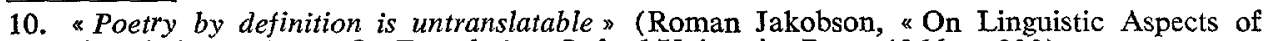
Translation », dans : On Translation, Oxford University Press, 1966, p. 238).

11. "Only creative transposition is possible" (ibid.). 
français, ait une valeur en soi. On pourrait bâtir, construire un texte avec le passif anglais, la forme du passif au centre. On pourrait également construire un texte où l'emploi du mot on en soi est essentiel pour la valeur de ce texte. Ce texte aurait alors comme base et comme élément important non seulement une idée, un message, une communication, mais une forme, avec on ou avec le passif. Et dans ce cas-là, parce qu'il s'agit non plus d'un phénomène humain, mais d'un phénomène ou anglais ou français, on ne pourrait plus traduire dans le sens normal mais métatraduire, c'est-à-dire expliquer ce jeu lingujstique parce que, dans toute langue, il existe la possibilité de créer des phénomènes de cette sorte, de jouer avec la langue, c'est-à-dire ne pas traduire directement, mais traduire indirectement, ce qui équivaut à commenter très souvent par la voie de la comparaison.

$M$. Gates : Il me semble que votre exemple de l'emploi de on est très intéressant. Mettons par exemple que nous construisions en français un texte sur une révolution où l'on peut employer le pronom on avec un fort sens affectif. Par exemple, on décrit la foule dans les rues :
"On hurle, on crie, on se dirige vers le palais, on détruit tout ». Là, je dirais que " on » a un sens fort affectif. Est-ce qu'il est possible de le traduire directement dans une autre langue ?

Réponse : Pour prendre l'exemple avec la répétition du mot «on» dans la description d'une foule, je pense qu'il serait peut-être possible de trouver une traduction directe en allemand à l'aide du mot man, mais il faudrait considérer tout le contexte. Mais on pourrait trouver un autre exemple, par exemple un texte français où " on " n'est pas seulement répété pour décrire une foule mais où le mot " on » est employé à des fins différentes pour désigner la $1^{\text {re }}$ personne; la $3^{\text {e }}$ personne, avec une pointe d'ironie, est substituée à nous, 1 re personne du pluriel, plus ou moins sans effet stylistique; mais dans l'ensemble il y aurait une concentration du mot « on " qui exploite toutes les possibilités qu'a le mot « on " et qu'il peut avoir seulement en français. Dans ces cas-là, il est tout à fait impossible de donner une traduction directe. Il faudrait se contenter d'un essai pour faire comprendre le mécanisme. 